International Journal of Building Pathology and Adaptation

emerald

PUBLISHING

International Journal of Building Pathology and Adaptation

The Consideration of Trees in Rights of Light Cases Part 2

\begin{tabular}{|r|l|}
\hline Journal: & International Journal of Building Pathology and Adaptation \\
\hline Manuscript ID & IJBPA-11-2017-0054.R3 \\
\hline Manuscript Type: & Original Article \\
\hline Keywords: & $\begin{array}{l}\text { Rights to Light, Point Loud, Crown Transparency, Measurement of Trees, } \\
\text { Software }\end{array}$ \\
\hline
\end{tabular}

SCHOLARONE ${ }^{m}$

Manuscripts 


\title{
The Consideration of Trees in Rights of Light Cases Part 2
}

\author{
Abstract \\ Purpose
}

This paper continues the research set out in The Consideration of Trees in Rights of Light Cases Part 1 , to consider whether it is possible to measure a tree or trees with sufficient accuracy that the impact on daylight within a building can be predicted in a way that can be applied in rights to light cases.

\section{Design/ methodology/ approach}

By reading published research on tree growth rates, crown transparency and theoretical modelling it is intended to determine the available methodologies for predicting light transmission through tree crowns, $t$. Then by inspecting common oak trees in all parts of the country, it is intended to review whether the available methodologies are capable of producing a relatively accurate result using manual methods or whether it is necessary to devise a software solution.

\section{Findings}

The research found that whilst theoretical methods exist for predicting light obstruction by trees, these could not be used in practice and that manual methodologies would not provide sufficient accuracy. However survey techniques such as 3D Point Cloud can be taken further with the development of a software solution that uses an algorithm to predict branch size and location where these are not visible in a survey.

\section{Practical implications}

This research concentrates on the theoretical aspect of assessing trees in rights to light cases. It is usually necessary for a live legal case to occur before research into software takes place and the conclusion reached was that it will be necessary to develop the software and then test survey a tree in full leaf and in the absence of foliage to determine whether an algorithm is sufficiently accurate and this will take time. It has also been demonstrated that trees may be considered as existing obstructions in rights to light cases and that once software has been developed and tested then it will enable developers to show that their proposals have less impact on the daylight within an adjoining property than would be the case if trees are ignored.

In many instances the economic development of a site, especially social housing, is limited by the rights to light of adjoining owners. Where it can be shown that the light levels enjoyed by those owners are already impaired by existing trees then this may assist the developer.

\section{Originality/value}

At the time of writing this paper there has been no published research to the extent contained in this paper.

Key words Rights to Light, Measurement of Trees, Point Cloud, Crown Transparency, Software.

Paper Type Research paper 


\section{Introduction}

In the previous paper "The Consideration of Trees in Rights to Light Cases Part1", the case was made that trees have been ignored in rights of light cases for what were, ostensibly, practical reasons and that there is now no legal or practical reason why this practice should continue. This view point is supported by Cooper R (2015) in his paper entitled "Easements: The Right to Light and Trees" where it was argued that the use of modern technology makes it possible to accurately assess the impact of trees on daylight to a room in existing circumstances and that one of the implications of this could be that the effective damage that would be caused by a proposed development might be reduced significantly.

The aim of this paper is to consider whether it is possible to measure a tree or trees with sufficient accuracy that the impact on daylight within a building can be predicted in a way that can be applied in rights to light cases.

This paper builds on the previous by investigating the following key factors:

1. Whether the measurement of the obstruction caused by trees can be standardised. This is relevant because without a standard approach it would not be possible for the parties or a court to be satisfied as to the relative accuracy of any results.

2. Verification of standard tree crown transparency factors. The existing published results which are quoted by BRE amongst others have not been considered in the context of rights to light and are used as a theoretical adjustment factor in daylight for planning considerations. The calculations for planning purposes differ significantly from those for rights of light.

3. Whether annual tree growth rates can be confirmed. This paper explains below that it is necessary to consider the obstruction caused by the tree(s) at a date one year prior to the actual measurement and thus it is necessary to be able to evaluate the growth that will have occurred in the preceding year and to deduct this from any calculations.

4. Comparison of results produced by point cloud survey with predictions using the above. For the purposes of advising a court it is essential that any theoretical calculations can be verified through actual measurement.

5. Development of software solutions. Ultimately any rights of light practitioner will need to have a tool that handles the necessary calculations and since most now use software based upon AutoCAD or other similar products it is necessary to ascertain whether it is possible to develop a software tool that will meet this requirement.

It was established in part 1 that, in order to achieve a valid result, it is necessary to be able to measure the minimum obstruction provided by a tree or trees during the year preceding the date of assessment. The minimum level of obstruction relates to the fact that the law only recognises a permanent obstruction. In Presland $v$ Bingham (1889) 41 ChD 268, it was found that a fluctuating obstruction such as packing cases may not be an interruption. In addition, in Bennison v Cartwright (1864) 5 B\&S 1, it was found that the dominant owner had to be aware of and to acquiesce in the obstruction for at least one year prior to any action to constitute a valid obstruction within the meaning of the law.

If it is accepted that trees and specifically deciduous trees in this instance, can constitute a permanent obstruction and that a dominant owner, by failing to take any action, is deemed to have acquiesced in that obstruction then it is necessary to be able to assess, accurately, the extent of that obstruction. However, it is recognised that no two trees are identical and therefore it may be necessary, if possible, to devise a methodology for the assessment of any tree or trees that may be applied from first principles.

Coniferous and other evergreen trees have limited transparency and significant variations in growth rates but, in terms of injury to the right to light, can largely be assessed by allowing 
solely for the growth that may have occurred within the year prior to assessment. Deciduous trees have significant variations in transparency as well as varying growth rates. Fortunately the measurement of transparency is only necessary for the bare branch condition but, to provide a basis for assessment at any time of the year it is necessary to understand the relationship between the crown size in full leaf and the transparency of the crown without of leaves.

\section{Methodology}

It is recognised that the organic nature of trees create wide varieties of shape and size as well as the differences between deciduous and evergreen trees. For the purposes of this research it has been decided to concentrate specifically on one common deciduous tree for which a considerable amount of research already exists in terms of measurement both of size and of daylight penetration.

To address the aim of this research it is necessary firstly to review the available research literature to establish how trees have been measured as a different baseline measurement could lead to a different result; how growth rates are predicted; how consistent is transparency data etc. From this it is possible to compare results with surveys undertaken on actual trees in various parts of the country in order to demonstrate validity.

Following on from this an attempt is made to manually model the obstruction to light within a property, where a tree is positioned in front of a window, comparing the result achieved with that which would be expected based upon existing research. Using photographs of trees and superimposing the outline of a window, a pixel count was undertaken to determine, as a ratio, how many pixels showed sky and how many were obscured by branches. This ratio was compared to the transparency ratios quoted in BR209 by BRE and others. Consideration is then given to whether the result is capable of replication if the same there is viewed from a different angle and also to multiple tree stands.

Finally consideration was given to the current state of technology for modelling trees with degrees of accuracy where the gaming world has made great strides in realistic constructions and may have algorithms capable of predicting branch locations even when a tree is in full leaf.

\section{Selection and Consideration of the English Oak (Quercus Robur)}

A number of tree types have been considered at various times of year but in order to formulate a base line methodology it has been decided to assess only one type of deciduous tree, the English Oak, against the following variables:

1. Annual growth rates at different ages of tree and assuming average growing conditions i.e. suitable soil and weather.

2. Method of measurement of trunk, crown, branch length etc.

3. Transparency data comparing winter with summer and across a range of tree ages.

4. How does one measure the transparency of a group of trees?

5. Mathematical difference if branches are assessed as individual obstructions or if the tree crown is consider as a solid of reduced dimensions.

Annual Growth Rates of Quercus Robur

Research by Rogers, Lawrence and Hutchings (2014) drawing on earlier research by McPherson et al gives average ring widths of $2.294 \mathrm{~mm}$ and a range of growth rates based on core samples taken over 64 years of between 0.0011 and $0.00317 \mathrm{~mm}$. In the context of rights of light calculations these amounts are very small and would probably not affect the results to a measurable extent. Their table 2 at page 77 shows an average tree canopy $(\mathrm{m})$ increment per 
year as 0.588 with a standard deviation of 0.43 but the point is made that growth rates are affected by the weather.

According to Gilman F and Watson D G (1994) Quercus Robur can grow to a height of 50 to 60 feet, a spread of 40 to 60 feet, the crown uniformity is a symmetrical canopy with a regular (or smooth) outline and, according to them, individuals have more of less identical crown forms. It is noted however that this is an American publication and growth rates and uniformity could be expected to vary through factors such as climate and soil conditions. In other gardening publications in the UK the ultimate height is given as 100 feet or 30 metres with average growth of 9 inches or 23 centimetres per annum and in a publication by Dover District Council entitled 'The English Oak' (2016) http://www.doverdistrictcouncil.com/the-english-oak/ It is said that the Oak tree, "has a period of quite rapid growth for around 80-120 years and that is followed by a gradual slowing down. By the time the tree is 80 years old, it may well be over 20 inches (50 centimetres) in diameter. Acorns are not produced until the tree is about 25-40 years old ....... and after about 250-350 years, the decline of the tree sets in, branches die back and the diameter growth slows right down".

In the same publication by Rogers, Lawrence and Hutching's, the average growth rates, plus standard deviation in brackets are given as average tree $\mathrm{DBH}$ increment in centimetres per annum is given as $0.228(0.8)$, the average tree height increment in metres per annum is given as $0.601(0.25)$ and the tree canopy increment in metres per annum is given as $0.588(0.43)$. This research has been added to by Monteiro $M$, Levanic $T$ and Doick K.J in their paper entitled 'growth rates of common urban trees in five cities in Great Britain' (2017).

From all of this it is apparent that, to be able to assess the probable growth rate, it is necessary to ascertain the approximate age of the tree in the first instance and to consider environmental factors that might affect growth. In addition the tree may have been cutback or have suffered die back but such things would be visible on inspection where they have occurred within the previous year.

In summary therefore it would be possible to estimate the amount of growth for a specific tree based upon its apparent age and condition.

Method of measurement of trunk, crown, branch length etc.

Trees are not lollipops and from a subjective perspective they do not usually have a uniform shape and therefore it is important to have a methodology for measurement of the various parts of the tree that is sufficiently accurate.

At its simplest this could be to measure the trunk from the ground to the lowest branch as being the height of the trunk and the average diameter as being the width. The crown would be the spread of the outermost branches for width and the height from exposed trunk at the base to the topmost branches and branch length would be the extent of any branch having a girth exceeding $150 \mathrm{~mm}$ measured from the trunk to the point at which the girth reduces below $150 \mathrm{~mm}$ and this is the methodology employed in much of the published research. The rationale for the $150 \mathrm{~mm}$ girth is twofold in that the diameter would be approximately $50 \mathrm{~mm}$ and below this value the branch would be unlikely to register as an obstruction and smaller diameters are more prone to movement caused by wind making them less than permanent in nature.

More accurate assessments, using computer aided design techniques, create a polyline around the tree crown but it must be recognised that the outline of a tree crown to any point in a room may differ between adjacent points and that therefore the greatest accuracy would be achieved by dealing with the tree in three dimensions rather than two as occurs with traditional manual assessments using a Waldram Diagram. 
Survey technologies have progressed by leaps and bounds since the time when the Waldram Diagrams were devised. The latest advancements include point cloud surveying which consists of a collection of data points defined by a given coordinates system. In a 3D coordinates system, for example, a point cloud may define the shape of some real or created physical system. Point clouds are used to create 3D meshes and other models used in 3D modeling for various fields including medical imaging, architecture etc. In a 3D Cartesian coordinates system, a point is identified by three coordinates that, taken together, correlate to a precise point in space relative to a point of origin. $X, y$ and $z$ axes extend in two directions and the coordinates identify the distance of the point from the intersection of the axes $(0)$ and the direction of divergence, expressed as + or - .

In the field, 3D scanners gather point measurements from real-world objects for a point cloud that can then be translated to a 3D mesh or NURBS or CAD model. Thus, using point cloud surveying it is now possible, in the right circumstances, to create an accurate three dimensional model of a tree at any point in time but, unfortunately, it is not always possible to use this technology when, for example, there is a need not to avoid requesting access to a neighbour's land or, where it is used, it may not be possible to determine, from the model, the extent of branches forming the crown or even the height of the trunk when the tree is in full leaf.

Various traditional methods of measurement exist and the three three basic parameters commonly measured to characterize the size of a single trunk tree are height, girth, and crown spread. A detailed guideline to these basic measurements is provided in The Tree Measuring Guidelines of the Eastern Native Tree Society by Will Blozan. According to Blozan "tree height is the vertical distance between the base of the tree and the highest sprig at the top of the tree. The base of the tree is measured for both height and girth as being the elevation at which the pith of the tree intersects the ground surface beneath, or "where the acorn sprouted."

When measuring tree heights from the ground the height can be calculated using the principle of similar triangles, a surveying technique taught to most scouts using a graduated pole. The second method uses a clinometer and tape and is commonly used in the forestry industry where the distance from the tree and angle of the clinometer are used to determine the height. Either of these two methods have the potential for significant errors including, for example, where the top of the tree is not visible from ground level.

The girth is a measurement is taken as the distance around the trunk of a tree measured perpendicular to the axis of the trunk and the use of girth to arrive at an equivalent diameter is an older forestry measurement that is still used. The measurement is commonly taken at around 1.5 metres above ground level. Where there are significant low branches below this height, ignoring any minor sprouts and dead branches, then the girth is measured at the narrowest point below the lowest branch.

Crown spread is a measurement of the footprint or plan area of the crown of the tree expressed as a diameter. Naturally the spread is non-uniform and so it is usual to measure perpendicular axes and to take the average of the two measurements to calculate the crown spread. One useful tool for reviewing crown spread and changes over time is to use Google Earth and the built in measurement tools.

Research by Frank (2010) established a methodology for plotting different tree shapes graphically using ternary plot diagrams (see Figure 1). Using this methodology he assessed the ratio of height to girth to average crown spread and plotted these on the chart below where he gave the measurement data for 140 live oaks measured as part of the NTS Live Oak Project. 


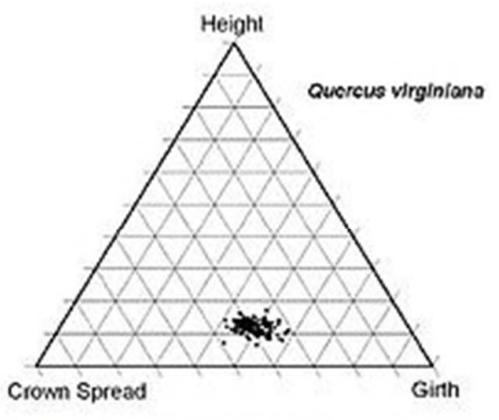

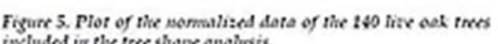
intuded in the trece shape analysis.

Figure 1 Tree shape diagram for live oak from Frank (2010)

This diagram shows a reasonably tight cluster and Frank reported that. "The height proportion exhibits a maximum of $17.23 \%$ of the shape value and a minimum of $6.55 \%$, the girth (minimum of 19 feet in the data set) exhibits a maximum of $58.25 \%$ and a minimum of $40.25 \%$, and Average Crown Spread maximum of $49.08 \%$ and a minimum of $30.92 \% . "$. It is noted that while the data set contains both multiple trunk trees and single trunk trees, both plot within the same tight cluster. It is possible therefore to conclude that any two measurements could be used to predict the third with a reasonable degree of accuracy.

By visiting a number of locations throughout the UK during 2015 to 2017 the author was able to verify the data to an acceptable degree of accuracy in healthy trees concluding that tree age and whether or not the tree had had branches trimmed at some point did not appear to affect the ratio to any significant degree. It was noted however that the transparency was increased, as would be expected, where branches had been thinned. Some trees that were assessed had suffered damage through storms etc. and these tended to sit outside the cluster.

In 2014 an assessment of the use of a hand-held camera for individual tree 3D mapping in forest sample plots was undertaken (Xinlian Liang, Anttoni Jaakkola, Yunsheng Wang, Juha Hyyppä, Eija Honkavaara, Jingbin Liu and Harri Kaartinen). It was established that using a terrestrial point cloud generated utilizing an uncalibrated hand-held consumer camera at a plot level and measuring the plot at an individual-tree level and then identifying individual tree stems in the plot and modelling these from the image-based point cloud and estimating the diameter-atbreast-height (DBH) of each tree, the detected-results could be compared with field measurements and with those derived from the single-scan terrestrial laser scanning (TLS) data. The experiment showed that the mapping accuracy was $88 \%$ and the root mean squared error of DBH estimates of individual trees was $2.39 \mathrm{~cm}$, which is acceptable for practical applications and was similar to the results achieved using TLS. The main advantages of the image-based point cloud data lie in the low cost of the equipment required for the data collection, the simple and fast field measurements and the automated data processing. The disadvantages of the image-based point cloud data include the limited capability of mapping small trees and complex forest stands.

In conclusion therefore, there are a number of ways in which the size of a tree may be assessed or estimated ranging from simple geometry and using the data referenced above, through to a full LiDAR/ point cloud survey. The level of accuracy will vary with methodology but this should be no worse than the levels of accuracy achieved using traditional Waldram methodologies. (Defoe, P.S. and Frame, I., 2005).

Transparency data comparing winter with summer and across a range of tree ages.

Data published by the BRE in BR209 does not specify whether the transparency varies with the age of the tree although research by Yates D, McKennan G T, 1989 and Wilkinson D M, Yates D 
and McKennan (1991) suggest that the data sets used are based upon trees in the 12-14 metre range. The important aspect is that any new methodology will require the ability to be able to measure a tree crown at any time of the year and to predict the transparency of that tree in its leafless condition for the purposes of assessing what the permanent obstruction would amount to.

The light attenuation for Quercus using their methods was given as $47.81 \pm 1.65$ in winter and between $82.35 \pm 2.05$ and $84.78 \pm 1.42$ in summer, whereas the BR209 gives the figures as transparencies of $20 \%$ in summer and $55 \%$ in winter although not all leaves had fallen. The research by Nowak D J (1996) mapping with a telescoping measuring rod at crown boundary points every 1.5 metres vertically and at every 45 degree azimuth radially to map the crown shape gave a shading factor for Quercus Robur of 0.81 in full leaf. On this basis it is reasonable to accept that, on average, the tree crown will intercept $45 \%$ of the light from the sky passing through the crown during the winter and around $80 \%$ when in full leaf, as a standard for assessment.

Bearing this in mind, a single tree may not block sufficient light, on a permanent basis, to significantly alter the so called 'grumble line' in a room which is currently set at $0.2 \%$ using the Waldram Diagram (Defoe, P.S. and Frame, I., 2005). However, it is quite common for trees to exist in groups and for this reason it is necessary therefore to consider how a group of trees might affect the light within a room where the trunk and/ or crowns overlap when viewed from within the room in the manner of the Waldram methodology.

\section{How does one measure the transparency of a group of trees?}

It may be perceived that a group of trees in close proximity would inevitably block more of the visible sky than a single tree. However Oak trees are rarely planted in uniform rows and the degree of overlap will vary considerably for a wide range of situations. In addition, the effect of perspective when the trees are viewed from worktop level within a room means that where trees are further from the view point their crown will be relatively lower and the value of obstruction based on the Waldram Diagram would be commensurately lower.

In the images below, to the left is a single tree and to the right the same tree viewed from a different aspect with other similar trees behind. The rectangles in each image represent windows of view from within a room and clearly demonstrate the difference in obstruction that results from several trees one behind the other. It also shows how the different perspective results in tree crowns further away from the viewpoint which appear lower and thus obscure sky visibility that would previously have been available either side of the trunk.

Where the crowns overlap the apparent transparency will reduce through the combined effect but there is no current published research on this and having compared numerous situations it is apparent that the number of variables is a significant problem. For example, the trees when viewed from a point within a room may be at different separations, be of different heights and different crown spreads as well as being in different alignments from any specific view point to the next. In this example, using a manual Waldram Diagram to assess the visible sky component, with the single tree acting as an obstruction, the sky component equates to approximately $3.3 \%$ of the sky dome. Whereas for the group the sky component equates to approximately $1.8 \%$ of the sky dome. Even the slightest change in viewpoint would produce a different result. 


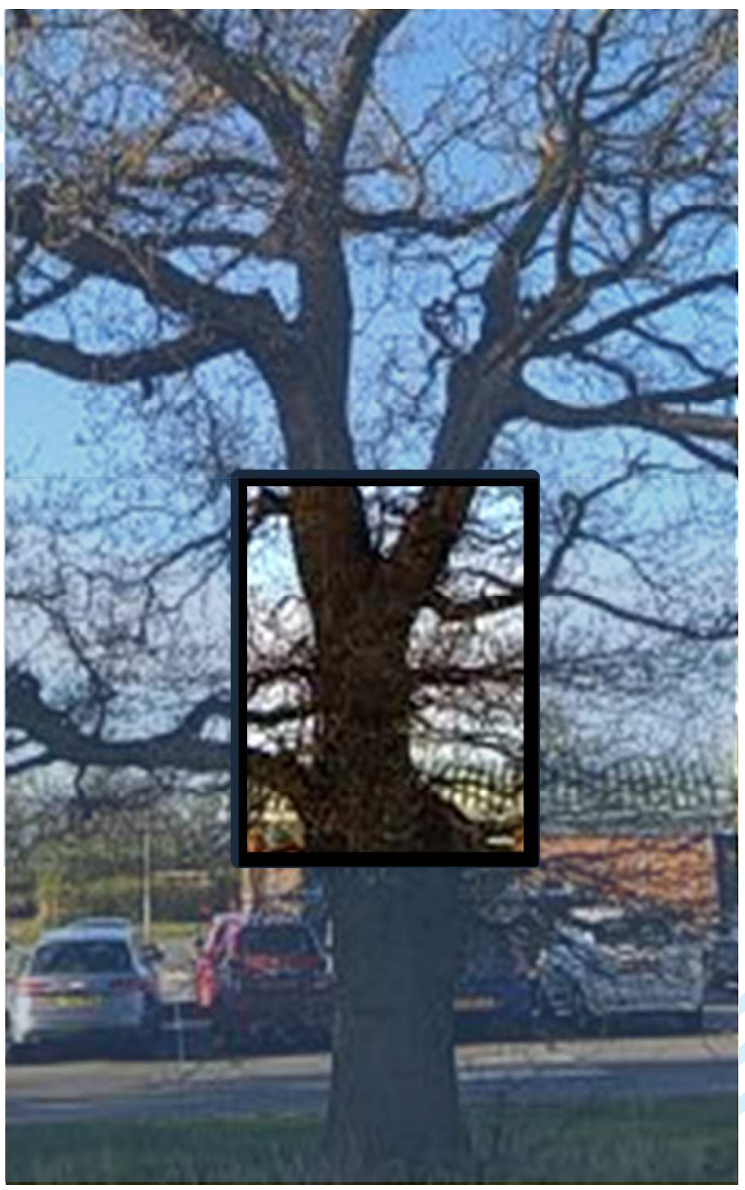

Figure $2 \mathrm{~A}$

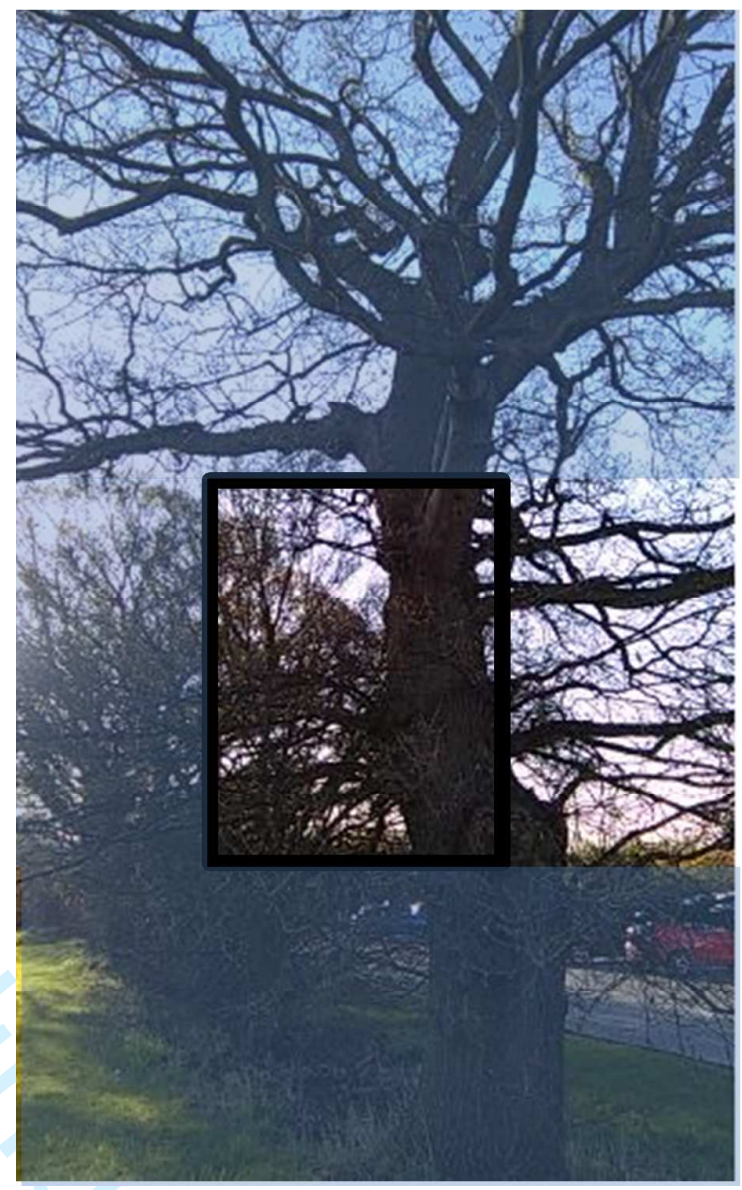

Figure 2B

It is not possible, therefore, to avoid the conclusion that it is only by accurate modelling, based on a point cloud survey, that it will be possible to accurately assess the combined impact of a stand of trees.

Mathematical difference if branches are assessed as individual obstructions or if the tree crown is considered as a solid of reduced dimensions.

It is important to understand whether, if it were possible to make manual assessments of trees as obstructions, there would be a mathematical difference between considering the tree crown as individual branch obstructions or as a solid based upon an assessment of the total volume of the branches. From a practitioner perspective it is often necessary to be able to simplify the modelling even when creating an electronic model but more especially when using traditional means.

The conclusion that has been reached regarding modelling of stands of trees would appear to preclude the use of manual methods in these instances but notwithstanding this it is necessary to consider whether a single tree might be modelled using traditional means and if so could this be achieved by converting the mass of branches into a single solid obstruction.

By using the tree image in figure $2 \mathrm{~A}$ and counting pixels as illustrated below in figure 3 , it is possible to estimate the sky visibility and obstruction. If the BRE transparency of $55 \%$ could be applied then pixel counting should show that $55 \%$ of the area of the image should be free from obstruction but, in fact, the pixel count shows that 1,025 out of 3,528 are unobstructed which equates to just over $29 \%$. 


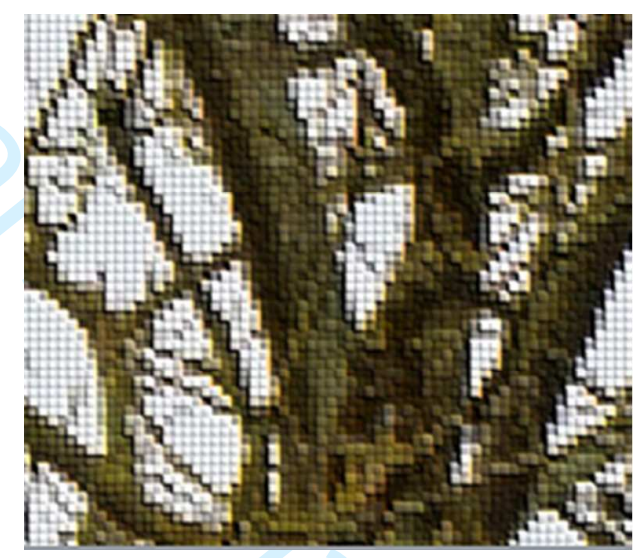

Figure 3 pixelated image of part of tree crown

This image was selected at random from a considerable number of photographs taken during winter months. The issue is that it concentrates on one section of the tree rather than the whole crown, as would happen when it is viewed through a window, and thus, whilst the tree crown, as a whole, may approximate to the BRE guidance in terms of transparency, the actual transparency when viewed from within a room will depend upon the part of the tree that is visible from any specific point, through the window.

Therefore, from a practitioner perspective, it will be necessary to firstly take an image such as the above and consolidate the branch obstruction into one mass, but this again raises another issue. Where on the diagram should the mass be positioned and what shape would be appropriate? In fact, the number of variable is too great to create as useable algorithm. The Waldram type diagram gives different values to each part of the diagram and so, for example, bringing the mass of the branches to each side into the centre would change the value of the obstruction. It is not possible to come to any conclusion other than that it is not possible to model a tree crown with any acceptable level of accuracy other than by using the electronic survey techniques described above.

\section{Development of software solutions}

This paper has demonstrated that it is possible to measure a tree or trees in terms of height and crown spread and to estimate with a reasonable degree of accuracy what the growth will have been in the previous year, but it has also demonstrated that manual methods of assessment of the light obstruction by trees cannot be undertaken with any degree of accuracy. However, survey techniques using 3D Laser Point Cloud can produce an accurate model that may be used within a computer programme that assesses light injury within the AutoCAD or REVIT environment (other software does exist but is not yet commercially available). The issue to be resolved is that the survey may be undertaken at any point during the year when a potential cause of action arises but for the purposes of rights to light calculations the model should be based upon the least foliage being on the tree, as previously explained.

Currently, when using a point cloud survey, the data is imported into AuoCAD and converted to a useable format for the model but there is no separate application that would allow the user to easily remove leaves from the tree model to expose the trunk and branches. Wang, Yinghui; Chang, Xin; Ning, Xiaojuan; Zhang, Jiulong; Shi, Zhenghao (2013) in describing how trees can be modelled in virtual reality, have described how it may be possible to effectively reverse engineer a tree in leaf to one without leaves.

They identified Nine sub-problems to describe the issues and their corresponding approaches.

3D laser point cloud reorganization; 
(2) tree segmentation or classification;

(3) skeleton point connection of discontinuous branches caused by occlusion from other branches or leaves;

(4) twigs skeleton synthesis;

(5) skeleton estimation of completely occluded branches;

(6) branch cross section radius calculation;

(7) leaf shape, foliage location and density estimation;

(8) phyllotaxis reconstruction;

(9) branch ridges and collars reconstruction.

For the purposes of rights to light it is only necessary to consider 1, 3, 5 and 6.

Reorganisation

Wang et Al stated that point cloud data can contain tens of billions of discrete scattered points and that the segmentation algorithm from such a large amount of data is time consuming and thus the need for reorganisation is inevitable. They identified that, whilst more research is required, the use of the multidimensional binary search tree i.e. k-d tree (Bentley 1975) could be developed.

\section{Skeleton Construction}

This process consists of two parts, firstly the skeleton extraction itself and secondly the branch radius estimation. In reviewing trees without leaves they concluded that the whole point cloud of branch surface is necessary when using level sets and thus the scan should be from multiple angles. Continuing to look at trees with partial occlusion by leaves they described how major branches could still be visible in part but that twigs and smaller branches would be inadequately sampled in the survey and, so they suggested that triangle similarity might be used. In addition, they examined the benefit of angle range judgement and particle flows to estimate the skeleton and found that the particle flow trace using two images taken from different angles of a given tree produced a reasonable representation.

Where trees are in full leaf the crown is obscured but they argue that, whichever way it grows, its surface is constructed by cloud in the form of a cylinder and the algorithm of the middle axis proposed by Marek et al (1998) to form the middle line of the crown surface could be sued to construct the skeleton.

Branch Radius Estimation

Leonardo Da Vinci surmised in the $15^{\text {th }}$ century that all the branches of a tree at every stage in its height, when put together, are of equal thickness to the trunk. This was supported much later by Shonzaki and West in their discussion of the pipe model and then proposed the WBE model (West 1999). The WBE model is a fractal like network that describes the vessels and tracheid between main and subsidiary branches. They further described how the relationship between branches could be predicted by assuming that the ratio between the child and parent radii is taken to be proportional to the ratio of the supported lengths for the child and parent raised to the $3 / 2$ power or that the ratio between one of the children and parent radii is taken to be proportional to the ratio of supported lengths for the child and the sum of all the children raised to the $1 / 2.49$ power.

When using these concepts for the purposes of rights to light calculations it is apparent that with a full multi point survey, and from the evidence demonstrated in the appended photographs (see appendix one) that it will be possible to pick up parts of branches when a tree 
is in full leaf and using an algorithm based upon the concepts described above, to extrapolate the likely branch layout with a reasonable degree of confidence. Smaller twigs are not so critical as they will make little mathematical difference to the resulting sky visibility.

\section{Conclusion}

In this second paper the aim was to investigate the following:

1. Whether the measurement of the obstruction caused by trees can be standardised

2. Verification of standard tree crown transparency factors.

3. Whether annual tree growth rates can be confirmed.

4. Comparison of results produced by point cloud survey with predictions using the above.

5. Development of software solutions.

Whether the measurement of the obstruction caused by trees can be standardised

It is an accepted fact that the courts will weigh the advice of any expert in the field of rights to light, on the basis of agreement between experts as to the methodology of measurement and to this end it will require a standardised approach in exactly the way that occurred through the original work of Percy Waldram (1909b).

In order to understand whether it is possible to have a standardised approach to measurement, it was decided to, so far as was possible, research a single tree type (Quercus Robur) and to assess whether the following variables could be defined sufficiently that a computer programme could be used to predict the impact on daylight within a room:

1. Annual growth rates at different ages of tree and assuming average growing conditions i.e. suitable soil and weather.

2. Method of measurement of trunk, crown, branch length etc.

3. Transparency data comparing winter with summer and across a range of tree ages.

4. How does one measure the transparency of a group of trees?

5. Mathematical difference if branches are assessed as individual obstructions or if the tree crown is considered as a solid of reduced dimensions.

This research has discovered that growth rates are reasonably predictable but have little impact on the final results; similarly, the methods of measurement of the parts of tree have already been standardised for other purposes. Transparency data has been shown to be of little use as it is not possible confirm that the transparency ratio works for just the part of the tree that is visible from within the room and thus, where a stand of trees is concerned, the results would be even more unreliable. It has also been demonstrated that converting the mass of branches to a single mass for calculation purposes would not be a practical solution.

On this basis it is necessary, if one wishes to achieve a standard methodology for measurement to consider how this may be approached using modern technology.

Verification of standard tree crown transparency factors.

The existing published results, which are quoted by BRE amongst others, have not been considered in the context of rights to light and are used as a theoretical adjustment factor in daylight for planning considerations. The calculations for planning purposes differ significantly from those for rights of light and to this end it was necessary to understand whether the transparency factors are sufficiently close to reality and whether they may be used for rights to light purposes albeit by using different algorithms for the final analysis.

What this research discovered was that the transparency factors may be correct when considering a whole tree but that they cannot be used when considering just the part(s) of a 
tree that may be visible through a window. Thus, even when using modern technology, it would probably not be possible to apply a single adjustment factor to any algorithm.

\section{Whether annual tree growth rates can be confirmed.}

It has been explained that it is necessary to consider the obstruction caused by the tree(s) at a date one year prior to the actual measurement and thus it is necessary to be able to evaluate the growth that will have occurred in the preceding year and to deduct this from any calculations.

From extensive research with the Forestry Commission and others it has been possible to confirm that growth rates are predictable and within a relatively small range and, in fact, this research has demonstrated that the growth rate of Quercus Robur is sufficiently slow that the changes in any one year are probably insufficient to affect the results. In this regard, any modern technology would probably not require an adjustment factor unless considering a tree type that has a greater growth rate as occurs with some coniferous trees.

Comparison of results produced by point cloud survey with predictions using the above.

For the purposes of advising a court it is essential that any theoretical calculations can be verified through actual measurement. One of the main difficulties associated with tree measurement is that, for rights of light purposes, it is necessary to assess the tree with minimum foliage, but the timing of any assessment might not coincide with this condition. It has been shown that published data on tree crown transparency is not reliable for this type of calculation and it has been demonstrated that it is not possible to equate the mass of individual branches with a single mass as the Waldram Methodology weights an obstruction according to the position of that obstruction on the diagram.

This research has shown, from a theoretical perspective, that the obstruction values could be assessed manually e.g. by using photographs to assess where the branches are and drawing up individual Waldram diagrams from a series of viewpoints within the room, but that, from a practical perspective, this would prove far too labour intensive to be economical. By comparison, it is possible to undertake the survey of the tree(s) using point cloud technology and that when the survey is undertaken with the tree(s) in minimum foliage conditions the results would be expected to have measurably greater accuracy.

The weakness that exists is in the timing because when a tree is in full leaf the point cloud survey will produce a complex set of data that would have to be interpreted so that the leaves are removed from the final model and whilst this could be done manually it would, at present, be very labour intensive. A software solution is therefore very desirable.

Development of software solutions.

Ultimately any rights of light practitioner will need to have a tool that handles the necessary calculations and since most now use software based upon AutoCAD or other comparable products it is necessary to ascertain whether it is possible to develop a software tool that will meet this requirement and to this end it appears possible that a software solution may be viable in the near future. The accuracy of any output from the software will depend upon the ability to undertake a full point cloud survey from as many directions as possible and, if the survey is undertaken whilst the tree or trees are in leaf then an algorithm will be required that takes the available evidence and extrapolates branch size and location whilst removing the foliage from the data.

The challenge will be for the software providers to develop the solution in the absence of a driver such as a live case requiring the assessment. 
Bibiliography

Anstey, J., 1992 Rights of Light and How to Deal With Them. RICS Books

Barrell Tree Consultancy, 2004 Trees and light; Arboriculture emerging from the shadows! Article in essential ARB Issue $12 \mathrm{BTC} / 16 / 2004$

Bickford-Smith S. and Francis A., 2000 Rights of Light, The Modern Law. Jordan Publishing Limited

Blozan Will, 2004 Tree Measuring Guidelines of the Eastern Native Tree Society Prepared by Will Blozan, ENTS President, October 2004

Revised March 2008

BRE Report (BR 209), 2011 Site Layout Planning for Daylight and Sunlight, a Guide to Good Practice, BRE

Chynoweth,P., 2004. Progressing the rights to light debate Part 1: a review of current practice. Structural Survey, 22(3), pp. 131-137.

Chynoweth,P., 2005. Progressing the rights to light debate: Part 2: the grumble point revisited. Structural Survey, 23(4), pp. 251-264.

Defoe, P.S. and Frame, I., 2005. The Validity of Daylight Calculations in Rights to Light, ed. In: $5^{\text {th }}$ International Postgraduate Research Conference, Manchester: The University of Salford 948957.

Defoe, P.S., 2007a. Was Waldram Wrong? [Online]. (last update Jan 2007) Available at: www.rics.org.uk [Accessed February 1, 2007].

Defoe, P.S. and Frame, I., 2007b. Was Waldram Wrong? Structural Survey, Vol 25, No2 pp98116(19) .

Defoe P.S. 2008a. The Validity of Daylight Calculations in Rights of Light Cases, Thesis Anglia Ruskin University.

Defoe P.S. 2008b. Light, How Much is Right, Building Services Journal 10/08 pp54-55

Defoe P.S. 2009 Waldram Was Wrong! Structural Survey, Vol 27, No3 pp186-199

Defoe P.S. and Frame I., (2010) The Validity of Daylight Calculations in Rights of Light Cases, COBRA conference Paris

Defoe P.S. 2011. Seeing the Light Article in The Land Journal p14 - 15, RICS

Defoe P.S., 2013. The Assessment of Daylight in Rights of Light Cases, COBRA Conference Delhi.

Defoe P.S and Frame I. and Spence C., 2014 The Consideration of Trees in Rights of Light Cases Part 1. Structural Survey Vol 32, No 3 pp

Forestry Commission Information Note FCIN12 November 1998 accessed August 2014 http:www.forestry.gov.uk

Frank, Edward Forrest. January 23, 2010. A Numerical Method of Plotting Tree Shapes. Bulletin of the Eastern Native Tree Society, Volume 6, Issue 1, Winter 2011, pp. 2-8. http://www.nativetreesociety.org/bulletin/b6 1/B ENTS v06 01.pdf Accessed March 4, 2013.

Gilman F and Watson D G (1994) Fact Sheet ST-558 October 1994

Littlefair, P.J., 1984. Daylighting Design and Energy Conservation, Building Research Establishment/ Council for National Academic Awards. 
Maltby, D., 2013. Discussion on Point Cloud Methodology (Personal Conversation at MBS, 6 Nov 2013)

Marek Teichmann and Seth Teller. Assisted articulation of closed polygonal models. In ACM SIGGRAPH 98 Conference abstracts and applications, SIGGRAPH ( 1998), pp 254.

Monteiro M. V., Levanic T., Doick K.J. 2017 Growth rates of common urban trees in five cities in Great Britain; a dendrochronological evaluation with an emphasis on the impact of climate; Urban Forestry and Greening 22 (2017) 11-23; Elsevier

Nowak D.J. 1996 Estimating leaf area and leaf biomass of open grown deciduous urban trees. Forest Science, 42 pp 504-507

Parratt, D., 2013 www.davidparratt.com/page 73.htm/ Accessed October 2013

Pitts, M., 2000. The grumble point: is it still worth the candle? Structural Survey, 18(5), pp. 255258.

Redler, A., 2013 Email conversation on Trees in Rights of Light Cases (Personal Conversation, 21 Dec 2013)

Rogers, Lawrence and Hutchings (2014); Determining Tree Growth in the Urban Forest; parallel session 1b: Urban Climate and Tree Growth.

USDA www.fs.fed.us/publications accessed December 2013

Waldram,P.J., 1909a. A Standard of Daylight Illumination of Interiors. The Illuminating Engineer, 3, pp. 469-470-472.

Waldram,P.J., 1909b. The Measurement of Illumination; Daylight and Artificial: With Special Reference to Ancient Light Disputes. The Journal of the Society of Architects, 3, pp. 131-132-140.

Wilkinson D.M., Yates D, and Mckennan G.T., Light Attenuation Characteristics of Seven Common British Trees. Arboricultural Journal 1991, 15: 37-44

Xinlian Liang, Anttoni Jaakkola, Yunsheng Wang, Juha Hyyppä, Eija Honkavaara, Jingbin Liu and Harri Kaartinen; The Use of a Hand-Held Camera for Individual Tree 3D Mapping in Forest Sample Plots, remote sensing ISSN 2072-4292.

Wang, Yinghui; Chang, Xin; Ning, Xiaojuan; Zhang, Jiulong; Shi, Zhenghao Tree Modeling Mehtods from 3D Point Cloud: A Survey. International Information Institute (Tokyo). Information ; Koganei Vol. 16, Iss. 6, (Jun 2013): 3675-3694

Yates D and McKennan G.T., Solar Architecture and Light Attenuation by Trees: conflict or compromise (2014); Arboricultural Journal 1989, 13:7-16.

Yoda K.Hozumi-K. Kira Shinozaki, . A quantitative analysis of plant form- the pipe model theory I. basic analyses. Japanese Journal of Ecology, 14, (1964), 97-105. [48] G. B. West, J. H. Brown, and B. J. Enquist. A general model for the structure and allometry of plant vascular systems. NATURE, 400(1999), 664-667.

\section{Statutes}

Prescription Act. 1832, (c71) England and Wales. England:

\section{Cases}

Ankerson v Connelly (1971) 1 Ch 678

W H Bailey \& Sons Limited v Holborn and Frascati Limited [1912] W 2399, [1914] 1 Ch 598

Colls v Home \& Colonial Stores (1904) A.C.179.

Lemon $v$ Webb [1894] 3 Ch 1; [1895] AC 1 
Metaxides v Adamson (1971) 219 EG 935

Presland v Bingham (1889) 41 ChD 268

Bennison v Cartwright (1864) B\&S 1

10

11

12

13

14

15

16

17

18

19

20

21

22

23

24

25

26

27

28

29

30

31

32

33

34

35

36

37

38

39

40

41

42

43

44

45

46

47

48

49

50

51

52

53

54

55

56

57

58

59

60 


\section{Appendix one}

Photographs of Trees showing how branch structure is visible even when trees are in in full leaf.
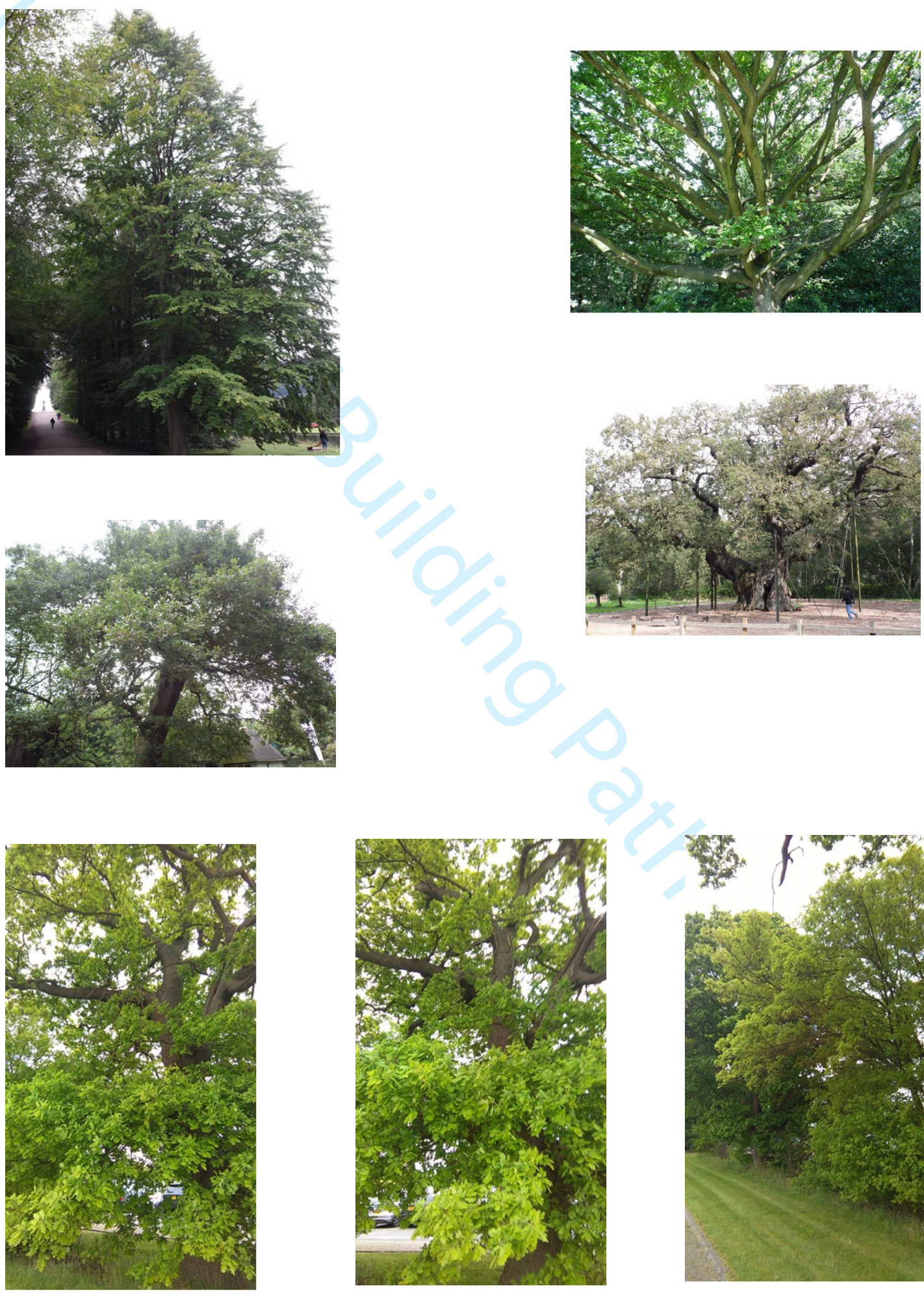

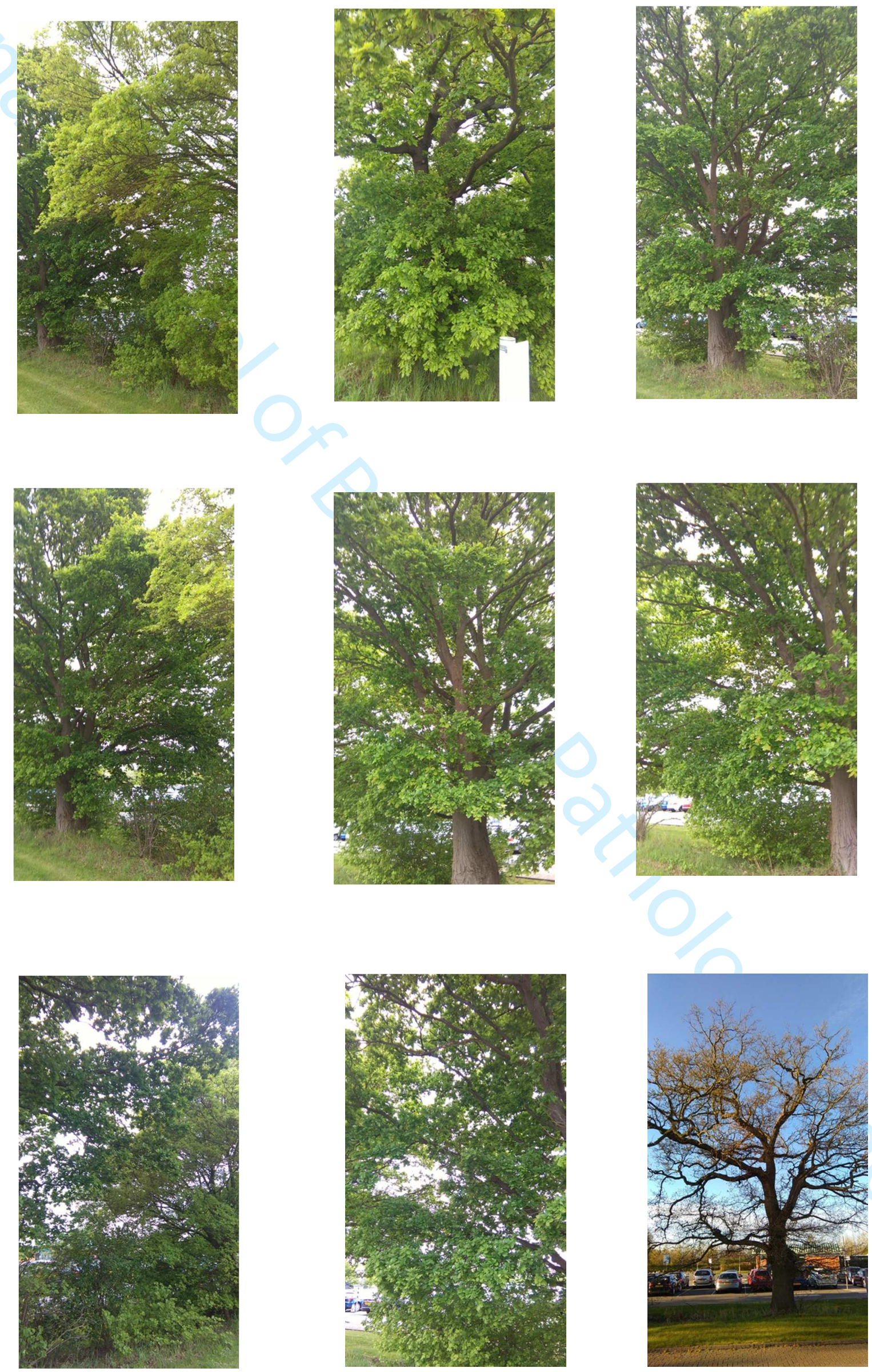